\title{
Search for anisotropic effects of hcp solid helium on optical lines of cesium impurities
}

\author{
M. Melich, ${ }^{1, \text { * } J . ~ D u p o n t-R o c, ~}{ }^{1}$ and Ph. Jacquier ${ }^{1}$ \\ ${ }^{1}$ Laboratoire Kastler Brossel, École Normale Supérieure; CNRS; UPMC; 24 rue Lhomond, Fr5005 Paris, France
}

\begin{abstract}
The anisotropic effect of a hcp ${ }^{4}$ He solid matrix on cesium atoms has been proposed as a tool to reveal the parity violating anapole moment of its nucleus. It should also result in splitting the $\mathrm{D}_{2}$ optical excitation line in a way depending on the light polarization. An experimental investigation has been set up using oriented hcp helium crystals in which cesium metal grains are embedded. Atoms are created by laser sputtering from this grains. Optical absorption spectra of the $\mathrm{D}_{2}$ line have been recorded in the temperature range of 1.0 to $1.4 \mathrm{~K}$ at liquid/solid coexistence pressure by monitoring the fluorescence on the $\mathrm{D}_{2}$ line at $950 \mathrm{~nm}$. No significant effect of the light polarization has been found, suggesting a statistically isotropic disordered solid environment for the cesium atoms.
\end{abstract}

PACS numbers: $67.80 . \mathrm{Mg}, 78.30 .-\mathrm{j}, 32.70 . \mathrm{Jz}, 32.80 . \mathrm{Ys}$

\section{INTRODUCTION}

Optical properties of heavy alkali metal atoms ( $\mathrm{Cs}, \mathrm{Rb}$ ) embedded in liquid or solid helium have been studied since 1990 [1] and reviewed in several articles [2]. Liquid helium and b.c.c solid behave as isotropic matrices for the atomic impurities. On the contrary, uniaxial h.c.p. solid is expected to perturb the atomic levels as a quadrupolar hamiltonian, lifting the degeneracy between Zeeman sublevels with different values of $|m|$. Such a feature was indeed observed experimentally in the two ground state hyperfine sublevels [3] and was interpreted as an anisotropic hyperfine interaction induced by the crystal. This effect was later proposed as a tool to reveal the parity violating anapole moment of the cesium nucleus [4]. An electric and a magnetic field with well defined orientations with respect to the helium crystal axis should produce a linear Stark effect, proportional to this anapole moment. This experiment requires a uniform crystal orientation throughout the sample. This remains to be achieved, since the experiments in the Cs ground state quoted above were interpreted as resulting from a statistically isotropic distribution of the local crystal c-axis. Also unknown is the perturbation of the local crystal order around each Cs atom. Optical absorption spectra are a sensitive tool to test the isotropy of the local environment of the alkali atoms in the solid matrix. We show that for an anisotropic matrix the shape of the $\mathrm{D}_{2}$ absorption line is expected to depend on the excitation light polarization, as well as that of the detected fluorescent light. In addition we report the result of an experimental investigation on cesium atoms sputtered in an initially mono-domain hcp crystal.

\section{CESIUM IMPURITY ABSORPTION SPECTRUM IN LIQUID AND SOLID HELIUM}

Optical transitions of a cesium atom embedded in a dense phase of helium have been described using various models [5]. Neglecting the hyperfine structure, the electronic energy levels reduce to the 8 sublevels shown in Fig. 1 . The $D_{1}$ line corresponding to the $6 \mathrm{~S}_{1 / 2} \rightarrow 6 \mathrm{P}_{1 / 2}$ transition is simply broaden by the interaction with the surrounding helium atoms. The properties of the $\mathrm{D}_{2}$ line $\left(6 \mathrm{~S}_{1 / 2} \rightarrow 6 \mathrm{P}_{3 / 2}\right.$ transition) is due to the removal of the 4 -fold degeneracy of the $6 \mathrm{P}_{3 / 2}$ level by any anisotropic environment, resulting into two Kramers doublets. Let us first ignore fluctuations

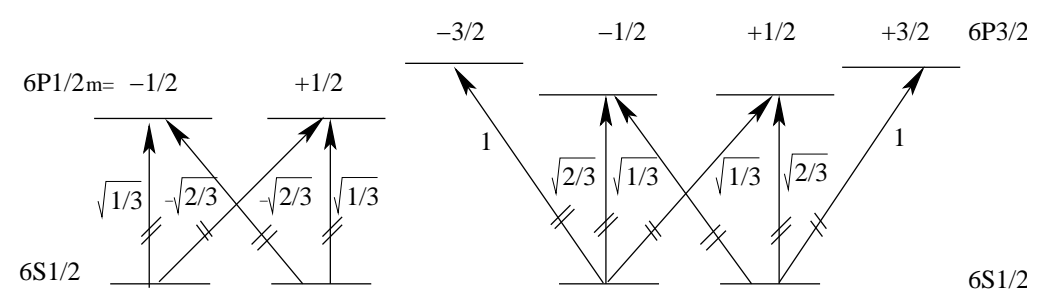

*Electronic address: mathieu.melich@lkb.ens.fr 
FIG. 1: Energy levels of cesium with a quadrupolar perturbation. Hyperfine structure is ignored. Numbers shown are proportional to the electric dipole matrix elements. The energy gaps between the various energy levels are not to the same scale. Ground state is duplicated for clarity.

and assume that the environment has an axial symmetry, as the hcp crystal does. Then taking the $z$-axis along this direction, its action on the $6 \mathrm{P}_{3 / 2}$ level may be written as $\mathcal{H}=A+B\left(3 J_{z}^{2}-J(J+1)\right) / 6$. The energy sublevels are the $m= \pm 3 / 2$ and $m= \pm 1 / 2$ magnetic sublevels with respect to the z-axis, with energy $A+B / 2$ and $A-B / 2$ respectively. The matrix elements of the electric dipole moment responsible for the optical transitions are reminded in Fig. 1. They imply that the weights of the two absorption line components strongly depend on the light polarization with respect to the $z$-axis. For z-polarized light, only the $|1 / 2|$ component is excited, while for $x$ - or $y$-polarization, the $3 / 2$ and $1 / 2$ components are excited with the probability $1 / 2$ and $1 / 6$ respectively. Experimentally, the absorption of light is very weak. Since radiative decay is the dominant decay process for the $6 \mathrm{P}$ excited state, one measures instead the intensity in the atomic fluorescence band as a function of the excitation wavelength. This intensity depends also on fluorescence polarization. Table I gives relative line intensities for various polarizations of fluorescence light. It is implicitly assumed that relaxation in the excited state prior to fluorescence preserve the populations of the $m$ sublevels. This amounts to neglect effects of the hyperfine coupling in the excited state. A fourth case is also considered which assumes on the contrary a complete depolarization of the excited state before fluorescence. It appears clearly that in any case the absorption spectrum strongly depends on excitation and detection polarizations. Consider now the

\begin{tabular}{|c|cc|cc|}
\hline Excitation & \multicolumn{2}{|c|}{ z-polarization } & \multicolumn{2}{|c|}{-polarization } \\
\hline \hline Fluorescence detection & $I_{3 / 2}$ & $I_{1 / 2}$ & $I_{3 / 2}$ & $I_{1 / 2}$ \\
z-analyzer & 0 & $4 / 9$ & 0 & $1 / 9$ \\
x-analyzer & 0 & $1 / 9$ & $1 / 4$ & $1 / 36$ \\
no analyzer & 0 & $5 / 9$ & $1 / 4$ & $5 / 36$ \\
depolarized & 0 & $4 / 9$ & $1 / 3$ & $1 / 9$ \\
\hline
\end{tabular}

TABLE I: Intensities of the two components $I_{3 / 2}$ and $I_{1 / 2}$ of the $\mathrm{D}_{2}$ absorption line excited by a beam on the x-axis and detected on the fluorescence intensity in the $\mathrm{y}$-direction for various polarizations.

effect of dynamic fluctuations. The effect of such a perturbation has been considered by Kinoshita et al. in the case of liquid helium [6], with no static anisotropy. Because of the three dimensions of space, an isotropic perturbation is less probable than anisotropic ones, and the line is split into two broad components of equal weights. Because of the statistical isotropy, no effect of polarization on the two component weights is expected. The more general case with a remaining static anisotropy is more complicated. It can be shown to reduce the contrast between the two components. If the static anisotropy is not small with respect to the fluctuations, some effect of light polarization should remain. This motivated the following experimental investigation.

\section{EXPERIMENTAL ARRANGEMENT AND PROCEDURE}

The experimental cell is a $4 \mathrm{~cm}$ stainless steel cube, with five silica windows (diam. $2.5 \mathrm{~cm}$ ), cooled from the top by a pumped helium- 4 fridge in the 1.0-1.4 K temperature range. To achieve single crystal growth, the nucleation and growth is made at constant temperature and pressure $(1.2 \mathrm{~K}$ and $25.5 \mathrm{bar})$. An electro-crystallization device 7] provides a unique seed which falls on the bottom of the cell. The crystal is subsequently grown from this seed. The orientation of the $c$-facet (most often horizontal) is easily monitored visually during the growth process which takes place below the corresponding roughning transition $1.3 \mathrm{~K} 8$ ]. Cesium atoms are produced in two steps by laser sputtering from a metal target [1] located in the upper part of the cell filled with superfluid. The laser pulses (1-5 mJ, $8 \mathrm{~ns}, \lambda=532 \mathrm{~nm}$ ) are provided by a Nd-YAG laser. The first step produces metallic grains, about $100 \mu \mathrm{m}$ in size, which fall on the crystal surface, when the interface is located in the middle of the windows. These grains are then incorporated into solid helium by subsequent growth. In the second step, the laser is focused on these grains, and atoms are produced in their immediate vicinity. This production phase lasts between a few minutes to half an hour at a rate of 2 pulses/second with the aim of maximizing the fluorescence signal on the $\mathrm{D}_{1}$ line. The $\mathrm{D}_{1}$ and $\mathrm{D}_{2}$ lines are excited by a tunable Ti:Sa CW laser scanned between 770 and $870 \mathrm{~nm}$, with a $z$ - or $y$ - polarization, the z-axis being along the vertical c-axis of the grown crystal. The fluorescence light in the $950 \mathrm{~nm}$ band [9, 10] is detected by a cooled APD and selected along the $y$-axis by a $900 \mathrm{~nm}$-edge filter which blocks the stray light from the laser. The other $880 \mathrm{~nm}$ band is also occasionally used to monitor the $\mathrm{D}_{1}$ fluorescence. 


\section{RESULTS}

A first observation is the time $T_{a}$ over which cesium atoms can be observed after their initial preparation. A very significant extension of $T_{a}$ was obtained when lowering temperature from $1.4 \mathrm{~K}$ to $1.0 \mathrm{~K}$. While at $1.4 \mathrm{~K}$, fluorescence from Cs atoms disappears in a few hours, as observed in Weis' group 11], at low temperatures it can be kept for days and even weeks. Because the diffusion of impurities in solid helium is expected to be thermally activated in this temperature range, such a behaviour is not unexpected. It is however of primary importance for future experiments with Cs atoms which will need long integration times.

Absorption spectra of the $\mathrm{D}_{2}$ line are shown in Fig. 2 for various polarizations of excitation and detection. A splitting of the line into two components is clearly visible. Its magnitude corresponds to an energy gap of $200 \pm 10$ $\mathrm{cm}^{-1}$. The line shape is similar to what has been reported for liquid helium [6] and solid helium at higher pressure [12]. Quantitatively, line widths are about 15\% larger than those in the liquid at 20 bar while splittings are $20 \%$ larger. The effect of the excitation polarization on the line shape is much smaller than expected. More precisely, the component weight ratio is 0.7 for $z$-excitation, $z$-analyzer and 0.85 for $y$-excitation, $x$-analyzer, to be compared with 0 and 9 expected respectively (see Table \).
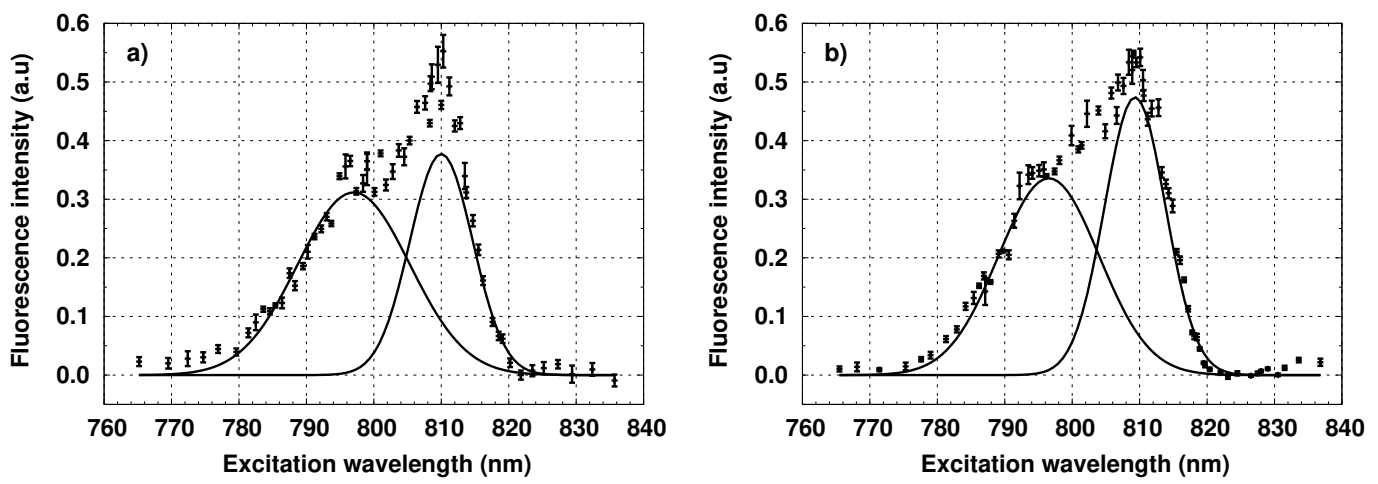

FIG. 2: Absorption spectra of the $\mathrm{D}_{2}$ line for different polarizations. (a) $z$-excitation, $z$-analyzer on detection, (b) $y$-excitation, $x$-analyzer on detection. Fitting the spectra with a sum of two gaussian lines yields the ones shown as plain curves.

\section{DISCUSSION}

There are two ways for explaining this result. The first one is to conclude that there is no permanent anisotropy in the environment of the atoms and that the splitting observed is mostly due to dynamic anisotropic fluctuations similar to those observed in liquid helium [6] as already discussed in reference [12]. The similarity of line shapes is indeed striking. Whether such an explanation is compatible with the hyperfine and magnetic resonance spectra observed in the ground state remains to be investigated. The second explanation is the one put forward in reference [3] : the sputtering process destroys the global order of the crystal which is transformed in a mixture of randomly oriented small hcp domains. Then no effect of the light polarization remains. If so, improvement of the crystal quality is to be searched for. Partial fusion, and re-crystallization from an ordered part of the solid is to be tried. It is unclear whether atoms will survive to such a process. Less damaging sputtering process using a femtosecond laser could also be attempted.

\section{Acknowledgments}

We acknowledge support from ANR, grant 05-BLAN-0084-01.

[1] A. Fujisaki, K. Sano, T. Kinoshita, Y. Takahashi, T. Yabuzaki, Phys. Rev. Lett. 71, 1039 (1993) ; M. Arndt, S.I. Kanorsky, A. Weis, T.W. Hänsch, Phys. Lett. A 174, 298 (1993). 
[2] B. Tabbert, H. Günther, G. zu Putlitz, J. Low Temp. Phys. 109, 653 (1997) ; S.I. Kanorsky, and A. Weis, Adv. in At. Mol. and Opt. Phys. 38, 121 (1997) ; P. Moroshkin, A. Hofer, S. Ulzega, and A. Weis, Low Temp. Phys. 32, 981 (2006).

[3] S. Kanorsky, S. Lang, T. Eichler, K. Winkler, A. Weis, Phys. Rev. Lett. 81, 401 (1998).

[4] M.A. Bouchiat, C. Bouchiat, Eur. Phys. J. D 15, 5 (2001).

[5] T. Kinoshita, K. Fukuda, Y. Takahashi, T. Yabuzaki, Z. Phys. B 98, 387 (1995) ; S.I. Kanorsky, A. Weis, R. Dziewior, T.W. Hänsch, Z. Phys. B 98, 371 (1995) ; J. Dupont-Roc, Z. Phys. B 98, 383 (1995) ; T. Nakatsukasa, K. Yabana, G.F. Bertsch, Phys. Rev. A 65, 032512 (2002) ; S.I. Kanorsky, M. Arndt, R. Dziewior, A. Weis, T.W. Hänsch, Phys. Rev. B 49, 3645 (1994).

[6] T. Kinoshita, K. Fukuda, T. Yabuzaki, Phys. Rev. B 54, 6600 (1996).

[7] K.O. Keshishev, A.Y. Parshin, A.V. Babkin, JETP 53, 362 (1981).

[8] S. Balibar, H. Alles, A.Y. Parshin, Rev. Mod. Phys. 77, 317 (2005)

[9] R. Müller-Siebert, Thesis, Université de Fribourg (CH), 2002.

[10] D. Nettels, A. Hofer, P. Moroshkin, R. Müller-Siebert, S. Ulzega, A. Weis, Phys. Rev. Lett. 94, 063001 (2005).

[11] M. Arndt, R. Dziewior, S.I. Kanorsky, A. Weis, T.W. Hänsch, Z. Phys. B 98, 377 (1995).

[12] P. Moroshkin, A. Hofer, D. Nettels, S. Ulzega, A. Weis, J. Chem. Phys. 124, 024511 (2006). 